\title{
Extractos de los documentos de posicionamientos y recomendaciones mexicanas en enfermedades cardiovasculares y COVID-19
}

\section{Excerpts from the documents of Mexican positions and recommendations in cardiovascular diseases and COVID-19}

Marco A. Alcocer-Gamba ${ }^{1 *}$, Pedro Gutiérrez-Fajardo², Alfredo Cabrera-Rayo ${ }^{3}$, Alejandro Sosa-Caballero ${ }^{4}$, Yigal Piña-Reyna ${ }^{5}$, José A. Merino-Rajme ${ }^{6}$, José A. Heredia-Delgado ${ }^{7}$, Jaime E. Cruz-Alvarado Jaime Galindo-Uribe ${ }^{9}$, Ulises Rogel-Martínez ${ }^{10}$, Jesús A. González-Hermosillo ${ }^{11}$, Nydia Ávila-Vanzzini ${ }^{12}$, Jesús A. Sánchez-Carranza ${ }^{13}$, Jorge H. Jímenez-Orozco ${ }^{14}$, Guillermo Sahagún-Sánchez ${ }^{15}$, Guillermo Fanghänel-Salmón ${ }^{16}$, Rosenberg Albores-Figueroa ${ }^{17}$, Raúl Carrillo-Esper ${ }^{18}$, Gustavo Reyes-Terán ${ }^{19}$, Jorge E. Cossio-Aranda', Gabriela Borrayo-Sánchez², Manuel Odín de los Ríos ${ }^{1}$, Ana C. Berni-Betancourt ${ }^{1}$, Jorge Cortés-Lawrenz ${ }^{1,5}$, José L. Leiva-Pons ${ }^{1,5}$, Patricio H. Ortiz-Fernández ${ }^{5}$, Julio López-Cuellar ${ }^{1,5}$, Diego Araiza-Garaygordobil', Alejandra Madrid-Miller ${ }^{2}$, Guillermo Saturno-Chiu², Octavio Beltrán-Nevárez², José M. Enciso-Muñoz ${ }^{16}$, Andrés García-Rincón ${ }^{5}$, Patricia Pérez-Soriano', Magali Herrera-Gomar', José J. Lozoya del Rosal', Armando I. Fajardo-Juárez', Sergio G. Olmos-Temois ${ }^{1}$, Humberto Rodríguez-Reyes ${ }^{1,2}$,

Fernando Ortiz-Galván ${ }^{1,2}$, Manlio F. Márquez-Murillo", Manuel de J. Celaya-Cota1, José A. Cigarroa-López'1,2, José A. Magaña-Serrano1,2, Amada Álvarez-Sangabriel'1, Vicente Ruíz-Ruíz', Adolfo Chávez-Mendoza1,2, Arturo Méndez-Ortíz¹, Salvador León-González¹, Carlos Guízar-Sánchez', Raúl Izaguirre-Ávila ${ }^{18}$, Flavio A. Grimaldo-Gómez ${ }^{18}$, Andrés Preciado-Anaya ${ }^{1,2}$, Edith Ruiz-Gastélum², Carlos L. Fernández-Barros², Antonio Gordillo², Jesús Alonso-Sánchez², Norma Cerón-Enríquez', Juan P. Núñez-Urquiza ${ }^{1}$, Jesús Silva-Torres ${ }^{1}$, Nancy Pacheco-Beltrán ${ }^{1}$, Marianna A. García-Saldivia', Juan C. Pérez-Gámez', Carlos Lezama-Urtecho ${ }^{7}$, Carlos López-Uribe7, Gerardo E. López-Mora ${ }^{11}$ y Romina Rivera-Reyes ${ }^{1}$

\footnotetext{
${ }^{1}$ Sociedad Mexicana de Cardiología; ${ }^{2}$ Asociación Nacional de Cardiólogos de México; ${ }^{3}$ Colegio de Medicina Interna de México; ${ }^{4}$ Sociedad Mexicana de Nutrición y Endocrinología; ${ }^{5}$ Sociedad de Cardiología Intervencionista de México; ${ }^{6}$ Asociación Nacional de Cardiólogos al Servicio de los Trabajadores del Estado; ${ }^{7}$ Sociedad Mexicana de Cirugía Cardiaca; ${ }^{8}$ Asociación Nacional de Cardiólogos del Centro Médico la Raza; ${ }^{9}$ Sociedad Mexicana de Medicina Crítica Cardiovascular; ${ }^{10}$ Sociedad Mexicana de Electrofisiología y Estimulación Cardiaca; ${ }^{11}$ Fundación Mexicana del Corazón; ${ }^{12}$ Sociedad Nacional de Ecocardiografía de México; ${ }^{13}$ Asociación de Fellows y Residentes Cardiólogos de México; ${ }^{14}$ Sociedad Mexicana Para el Cuidado del Corazón; ${ }^{15}$ Sociedad Mexicana de Ecocardiografía e Imagen Cardiovascular; ${ }^{16}$ Asociación Mexicana para la Prevención de la Ateroesclerosis y sus Complicaciones; ${ }^{17}$ Sociedad Mexicana de Anestesiólogos Cardiotorácicos; ${ }^{18}$ Sociedad Mexicana de Trombosis y Hemostasia; ${ }^{19}$ Comisión Coordinadora de Institutos Nacionales de Salud y Hospales de Alta Especialidad
}

\section{Correspondencia:}

*Marco A. Alcocer-Gamba

E-mail: marco.alcocerg@gmail.com
Disponible en internet: $26-05-2020$ Arch Cardiol Mex. 2020;90(Supl):100-110

www.archivoscardiologia.com 1405-9940/@ 2020 Instituto Nacional de Cardiología Ignacio Chávez. Publicado por Permanyer. Este es un artículo open access bajo la licencia CC BY-NC-ND (http://creativecommons.org/licenses/by-nc-nd/4.0/). 


\title{
Resumen
}

Se presentan las recomendaciones en las cuales la Sociedad Mexicana de Cardiología (SMC) en conjunto con la Asociación Nacional de Cardiólogos de México (ANCAM), así como diferentes asociaciones médicas mexicanas vinculadas con la cardiología, después de una revisión y análisis exhaustivo y consensuado sobre los tópicos relacionados con las enfermedades cardiovasculares en la pandemia de COVID-19, se analizan posturas científicas y se dan recomendaciones responsables sobre medidas generales a los pacientes, con cuidados personales, alimentación saludable, actividad física regular, acciones en caso de paro cardiorrespiratorio, la protección del paciente y del personal de salud así como las indicaciones precisas en el uso de la imagen cardiovascular no invasiva, la prescripción de medicamentos, cuidados en tópicos específicos como en la hipertensión arterial sistémica, insuficiencia cardiaca, arritmias y síndromes coronarios agudos, además de hacer énfasis en los procedimientos de electrofisiología, intervencionismo, cirugía cardiaca y en la rehabilitación cardiaca. El interés principal es brindar a la comunidad médica una orientación general sobre el quehacer en la práctica cotidiana y pacientes con enfermedades cardiovasculares en el escenario esta crisis epidemiológica sin precedentes de COVID-19.

Palabras clave: COVID-19. Cubrebocas. Paro cardiaco la pandemia. Hemodinamia.

\begin{abstract}
The recommendations in which the Mexican Society of Cardiology (SMC) in conjunction with the National Association of Cardiologists of Mexico (ANCAM) as well as different Mexican medical associations linked to cardiology are presented, after a comprehensive and consensual review and analysis of the topics related to cardiovascular diseases in the COVID-19 pandemic. Scientific positions are analyzed and responsible recommendations on general measures are given to patients, with personal care, healthy eating, regular physical activity, actions in case of cardio-respiratory arrest, protection of the patient and health personnel as well as precise indications in the use of non-invasive cardiovascular imaging, prescription of medications, care in specific topics such as systemic arterial hypertension, heart failure, arrhythmias and acute coronary syndromes, in addition to emphasizing electrophysiology, interventionism, cardiac surgery and in cardiac rehabilitation. The main interest is to provide the medical community with a general orientation on what to do in daily practice and patients with cardiovascular diseases in the setting of this unprecedented epidemiological crisis of COVID-19.
\end{abstract}

Key words: COVID-19. Mouthguards. Cardiac arrest pandemic. Hemodynamics.

\section{Recomendaciones generales}

En respuesta al aumento de casos con COVID-19, múltiples sociedades médicas en México y en el mundo hemos emitido recomendaciones tanto preventivas como terapéuticas. Todas coinciden en aplicar las medidas preventivas básicas que incluyen:

- Quedarse en casa, promover distanciamiento social minimo de 1.5 metros

- Lavarse frecuentemente las manos, al menos durante 20 segundos, con agua y jabón o utilizar alcohol gel.

- Evitar tocarse ojos, nariz y boca para evitar la autoinoculación. En caso de tos o estornudos cubrirse la nariz y boca con un pañuelo desechable o el ángulo interno del brazo (pliegue interno del codo) y lavarse las manos posteriormente.

En el interior de la casa es importante favorecer la iluminación y ventilación natural, se deben desinfectar superficies y objetos de uso común tanto en casa como en oficinas y transporte, asimismo tener hábitos alimenticios saludables con calidad y cantidad balanceada, evitando así el consumo abundante de azúcares y grasas saturadas. En caso de menor actividad física se debe disminuir la ingesta calórica para evitar aumento de peso, pero sobre todo se debe continuar con régimen de ejercicio físico aeróbico en la medida de lo posible.

Aquellos pacientes que reciben tratamiento farmacológico deben de continuarlo de acuerdo a la prescipción médica y no se recomienda suspenderlo por iniciativa propia. Se sugiere mantener comunicación por medios digitales con su médico tratante y si fuera necesario acudir a consulta presencial hacerlo preferentemente solo o máximo con un acompañante.

En caso de ser un paciente con un dispositivo implantado (DAl, marcapasos, o resincronizador), en un gran número de casos están conectados por telemetría (a distancia). En caso de notar una señal auditiva del dispositivo, contactar de manera telefónica a su hospital o médico responsable. En pacientes con diabetes mellitus, deberá mantener niveles de glucosa 
adecuados mediante el apego a su tratamiento, alimentación sana, ejercicio regular y monitoreo frecuente.

Si tiene algún síntoma sugestivo de la enfermedad por COVID-19, tales como fiebre $\left(>38^{\circ} \mathrm{C}\right)$, dolor de cabeza, dificultad respiratoria, cansansio, dolores musculares, dolor de garganta, escurrimiento nasal y tos seca o ha tenido contacto con alguna persona sospechosa o confirmada de COVID-19, comuníquese directamente con su médico, quien le indicará cuál es la mejor conducta a seguir. También se puede llamar al 8000044800 para atención inmediata y cumplir los protocolos establecidos por la Secretaría de Salud.

\section{Ejercicio y COVID-19}

Con relación a las medidas para cuidar la salud, el ejercicio es un tipo de actividad física cuyo propósito es generar o mantener una aptitud corporal adecuada, deber tener el concepto FITT, es decir, una frecuencia, intensidad, tipo y tiempo. Estos deben ser determinados por su médico tratante y que se adecuen a tu tolerancia al ejericio, acorde a su historial. Es ideal que el ejercicio se lleve a cabo en un lugar seguro, con luz y ventilación adecuadas y con ropa cómoda. De acuerdo a las recomendaciones de instituciones de salud y gobierno del lugar donde se radique, se prefiere durante esta contingencia de salud realizar ejercicio en la medida de lo posible dentro de casa. De no ser posible, o las recomendaciones de salud y gobierno local lo permitan; al realizar ejercicio fuera de casa, debe considerar la distancia al caminar en una misma dirección en línea recta y deberá haber separación entre las personas de 4-5 metros. Para aquellos que corren o realizan bicicleta a baja velocidad, la distancia de separación entre las personas de al menos 10 metros, y finalmente en aquellos quienes realizan bicicleta a alta velocidad, la distancia de separación deberá ser de al menos 20 metros.

Se sugiere seguir estos pasos:

La frecuencia del ejercicio aeróbico debe ser mínimo 30 minutos, la mayoría de los días de la semana, o bien por lo menos cinco días de la semana, lo que permitiría alcanzar un total de 150 minutos de ejercicio aeróbico de ligera a modera intensidad. En caso de no tolerar los 30 minutos, la sesión se puede fragmentar de acuerdo a la tolerancia. La intensidad del ejercicio en aquellos con factores de riesgo para enfermar del "corazón" o quienes ya tienen enfermedad cardiovascular manifiesta, se recomienda sea de ligera a moderada intensidad. El consejo práctico, corresponde a realizar ejercicio con la intensidad suficiente para "mantener una conversación mientras se realiza dicho ejercicio".

Tener presente que cada intervención con el ejercicio, debe ser con una dosis correcta, de manera que se obtengan los beneficios deseables y duraderos. Evitar la dosis excesiva de ejercicio, porque el sobre-entrenamiento puede provocar cansancio y pérdida de interés por el mismo.

Previo a cada sesión de ejercicio, debe haber una autoevaluación y considerar pautas de seguridad. No realizar ejercicio si existe fiebre, algún proceso de infección, cambio en la condición cardiovascular, ayuno prolongado. En este caso se sugiere una ingesta ligera de alimentos, o si de acuerdo a lo referido por el médico tratante, tiene algún dato de alarma.

Se sugiere apegarse a la fase de calentamiento, fase principal y estiramientos con las siguientes recomendaciones generales. Calentamiento: 5 a 10 minutos se puede hacer una caminata de intensidad ligera, o bien empezar con ejercicios de movilidad en las grandes articulaciones repitiéndolos 8 a 12 veces cada uno, iniciando desde la cabeza y terminando con pies. Ejercicios aeróbicos: Debe ser contínuo y rítmico durante el tiempo que tolere dicho ejercicio con movimiento de grandes grupos musculares. El ejercicio aeróbico es el que más beneficios brinda para la salud cardiovascular; como pérdida de peso, disminución de niveles de azúcar, colesterol y de la presión arterial. Ejemplos tangibles del ejercicio aeróbico son: nadar, trotar, caminar, andar en bicicleta y baile, entre otros. Lo anterior ejecturalo de acuerdo a preferencias, disponibilidad y tolerancia. Ejercicios de fuerza o "anaeróbicos": si bien, no serán los que más beneficios brindan a la salud cardiovascular, no se contraindican con una dosis correcta. Ayudan a ganar fuerza y masa muscular y brindan fortaleza a tu sistema músculo esquelético entre otros beneficios. Al ser ejercicios de mayor intensidad se sugieren periodos cortos de tiempo. Algunos ejemplos son abdominales, sentadillas, flexiones, plancha, levantamiento de pesas (menos de 5 libras). Se sugiere hacer desde 6 hasta 12 repeticiones de cada ejercicio, esto es una serie, y puedes hacer de 2 a 4 series. Debes coordinar el patrón de la respiración, de manera que al momento de hacer la fase concéntrica "de esfuerzo" en el ejercicio de fuerza debes exhalar y al disminuir el esfuerzo, debes inhalar. Estiramiento: consiste en relajar los músculos trabajados al final de su ejercicio para evitar contracturas y lesiones crónicas. Estos son ejercicios de estiramientos estáticos libres y se deben entre 8 y 12 segundos en cada posición. Es importante realizarlos de forma lenta y 
progresiva, respetando siempre el umbral de dolor combinándolos con el patrón de la respiración.

Tener presente que para el personal de salud y para la familia del paciente, la salud, el bienestar y su reintegración en actividades habituales de todo tipo con la consejería correcta, son lo más importante.

\section{Uso de cubrebocas y equipo de protección personal}

En esta pandemia es clave en un paciente con riesgo cardiovascular reducir la exposición social o a contactos de riesgo. El uso de un cubrebocas es útil para reducir la tasa de contagio de una enfermedad ya que los virus y secreciones producidos en la mucosa nasal y bucal, pueden ser expulsados al hablar, toser y estornudar hasta una distancia de 2 metros.

Los cubrebocas de TNT (Tela No Tejida), impermeables y caseros, son útiles durante la pandemia, usarlos CORRECTAMENTE, reduce el contagio y mitiga la duración de la pandemia pero su uso INCORRECTO puede ser perjudicial, que el no usarlo, al convertirlo en un objeto transmisor y dispersor del virus. Durante una pandemia, el uso del cubrebocas esta indicado a personas con o sin síntomas con igual importancia y rigor, de forma adicional agregar el uso de lentes que recubren el ojo de forma completa. En el caso del personal no médico con seguimientos previos necesarios deben usar siempre un cubrebocas de tela LIMPIO cubriendo boca y nariz. Una vez puesto el cubrebocas no debe de ser manipulado sino hasta el momento de quitarlo y no debe ser usado como diadema o collar pero en caso de llegar a tocarlo se deberá lavar las manos o usar gel con alcohol de forma inmediata. Retirarse el cubrebocas correctamente significa que no debe tocar la cara, ni sacudirlo y debe ser tirado a la basura si presenta daños. Los cubrebocas de tela impermeable deben lavarse diario o inmediatamente después de su uso, se recomienda etiquetar con nombre y no usarse por más de 2-3 horas, pasado este tiempo debe ser cambiado. Esta aparente pequeña protección, sumada millones de veces, asociada a las medidas de higiene ya implementadas, se vuelve una fuerza de protección masiva que podría llegar a ser significativa y definitoria en la evolución de la pandemia para la población de todo el país 1 .

En cuanto a la atención pre-hospitalaria el personal de salud debe ser notificado cuando se sospecha de COVID-19 en un paciente que requiere atención y traslado, idealmente debe contarse con equipo de protección personal (EPP) básico o completo, que comprende gafas, mascarilla N95, guantes, bata impermeable y botas para que en caso de atender a cualquier paciente con signos o síntomas de una infección respiratoria, tener presente que la evaluación debe ser a una distancia de al menos 1.5 metros del paciente, con contacto mínimo hasta que se le coloque mascarilla facial, en caso de realizar traslado del paciente solo debe estar el personal esencial para reducir el riesgo de contagio $^{2}$. El personal de atención pre-hospitalario solo debe llevar al enfermo a un hospital preparado para recibir casos con sospecha de COVID-19 y debe notificar a la institución a fin de que puedan tomar las precauciones de control antes de su llegada ${ }^{3}$. El conductor de la ambulancia debe estar aislado del compartimiento del paciente. En caso de usar un vehículo sin compartimiento del conductor aislado, se deben abrir las ventanas en el área del conductor y encender la ventilación en la parte posterior del vehículo al nivel más alto (esto crea un gradiente de presión negativa en el área contaminada) ${ }^{4}$.

En el área de urgencias se deben seguir las precauciones estándares, incluido utilizar un EPP completo, el paciente debe usar mascarilla facial para controlar la fuente, si tiene oxígeno por cánula, debe usar una mascarilla sobre ésta o utilizar mascarilla con oxígeno ${ }^{5}$. Los procedimientos que generan aerosoles (RCP e intubación endotraqueal), exponen al personal de salud a un riesgo alto de contagio por lo que idealmente se deben realizar en salas de aislamiento para "infecciones transmitidas por el aire". Todo el personal debe utilizar EPP. Solo los proveedores esenciales para la atención del paciente y el apoyo del procedimiento deben estar presentes. La habitación deberá limpiarse y desinfectarse después del procedimiento. Los casos sospechosos o confirmados con COVID-19 deben ser atendidos en una habitación individual con la puerta cerrada. Las salas de "infecciones transmitidas por el aire" se deben reservar para pacientes sometidos a procedimientos que generan aerosol. En caso de necesitar intubación endotraqueal, considere intubación de secuencia rápida con el EPP completo y de ser posible utilice videolaringoscopio para evitar mayor contacto con la cara y las secreciones del paciente ${ }^{6}$.

\section{Paro cardiorrespiratorio}

Si presencia un paro cardiaco extra-hospitalario:

a) NO compruebe la respiración, NO realice ventilación de boca a boca.

b) Si es posible use guantes y trate siempre de cubrir su boca y nariz (mascarilla). 
c) Use un desfibrilador automático (DEA) y siga las indicaciones que le proporcione.

d)En caso necesario solo realice compresiones torácicas.

e)Tras la reanimación, lávese las manos tan pronto como sea posible con agua y jabón.

Soporte vital básico por personal de atención pre-hospitalaria:

a)Si sabe que el paciente está en paro cardiaco, por lo menos un miembro del equipo, se debe colocar el EPP completo, durante el trayecto.

b) Todos los miembros del equipo deben utilizar siempre guantes, gafas protectoras, mascarilla 0 cubrebocas.

Uno de los miembros del equipo con EPP básico inicia las compresiones torácicas continuas, mientras el otro coloca el DEA. NO realizar ventilación con bolsa-válvula-mascarilla.

c) En los niños en paro cardiaco, la ventilación inicial es crucial: a pesar del riesgo de propagación del virus, realice 5 respiraciones iniciales con bolsa-válvula-mascarilla.

d)NO realizar RCP básica ni avanzada sin llevar la protección adecuada, iprotegerse ante la infección!

En unidades de Soporte Vital Cardiovascular Avanzado (SVCA) extra-hospitalaria el miembro del equipo responsable de la vía aérea colocará un dispositivo supra glótico (DSG), con un filtro entre éste y la bolsa de resucitación por lo que no se recomienda la intubación endotraqueal, la complejidad de la intubación aumenta sustancialmente el riesgo de contagio. Si cuenta con un dispositivo de compresión torácica mecánica, dos miembros del sistema de emergencia médico (SEM) equipados con EPP básico pueden colocar el compresor torácico.

Se debe realizar una revisión y organización del equipo previo a la llegada del paciente, asignando los roles de cada miembro y el flujo de procedimientos reducirá el riesgo de contaminación e infección del equipo.

En los Servicios de Urgencias, áreas de hospitalización, transferencia de pacientes dentro del hospital, etc. Se recomienda que el equipo de atención este compuesto del mínimo de personas necesarias para reducir el riesgo de contagio, cuatro personas es considerado un número apropiado y todos equipados con EPP completo.

Se recomienda personal de apoyo para labores de logística, para retiro de EPP o como personal de reserva.

a) En caso de un paro cardiaco, un miembro equipado con EPP básico (gafas, máscara, guantes y delantal) puede iniciar las compresiones torácicas continuas, mientras el resto del equipo se viste con un EPP completo. Una vez equipados con EPP completo, un miembro del equipo releva a su compañero en las compresiones torácicas para que este pueda equiparse.

b)Continúe la resucitación según el algoritmo estándar de la AHA.

c) Se hará cargo de la vía aérea la persona más experimentada en esta técnica. Se procede a intubación orotraqueal lo antes posible con un videolaringoscopio con un monitor separado que permita la mayor distancia del paciente. Si el primer intento de intubación fracasa, coloque un dispositivo supraglótico de segunda generación ("tubo laríngeo") evitar fugas para la aerosolización y coloque un filtro HEPA entre el tubo y la bolsa de resucitación.

d)NO ventilar con bolsa-válvula-mascarilla por el riesgo de salpicaduras que conlleva.

Si cuenta con un dispositivo de compresión torácica mecánica, dos miembros del equipo vestidos con EPP básico pueden colocar el compresor torácico y luego vestirse con un EPP completo para continuar con el algoritmo de atención.

\section{Estudios de diagnóstico no invasivo}

La realización de métodos diagnósticos de imagen cardiaca implica la potencial exposición al agente SARS-COV-2, hay que tener en cuenta el riesgo/beneficio de la realización de los mismos ${ }^{7}$; en el caso de ecocardiografía las recomendaciones para ecocardiograma durante esta pandemia protegiendo tanto al paciente como al personal de salud, resumiéndolas en lo siguiente:

1. El estudio debe ser solicitado solo por el personal experto.

2. Debe realizarse por médicos especialistas con la finalidad de evitar repetir estudios innecesarios.

3. El estudio solo debe realizarse en caso de cambiar sustancialmente el manejo del paciente.

4. Se debe considerar el riesgo de infección y evitar el abuso en el consumo del equipo de protección personal.

5. Los estudios de imagen en pacientes no urgentes deben considerarse cancelarlos o posponerlos.

6. Se recomiendan ecocardiogramas enfocados (FOCUS) a un objetivo determinado.

7. Se recomienda un equipo pequeño, de bolsillo fácil de manejar, de limpiar y de cubrir.

8. El estudio enfocado debe incluir: evaluación de la función sistólica ventricular izquierda, evaluar 
alteraciones regionales de la contractilidad, dimensión telediastólica, evaluación de la función sistólica del ventrículo derecho (TAPSE, cambio de la fracción de acortamiento de área, dimensión telediastólica), alteraciones valvulares por estimación visual y/o derrame pericárdico.

9. Omitir el registro del monitoreo eléctrico.

10. Los ecocardiogramas transesofágicos no están contraindicados, pero deben de evitarse en lo posible.

11. Los ecocardiogramas de esfuerzo con cicloergómetro deben evitarse en lo posible por la gran diseminación de virus, debe optarse por los estudios farmacológicos.

12. Las mediciones deben realizarse offline.

13. En el reporte debe hacerse referencia que el estudio fue restringido por la enfermedad COVID-198,9.

En relación a los estudios de tomografía, es recomendable que estudios no urgentes como el índice de calcio coronario, el estudio anatómico de cardiopatías congénitas o la evaluación de venas pulmonares, no se realicen por el momento ${ }^{10}$; de acuerdo a su urgencia, los estudios se clasifican en: electivos (realizar en 8 semanas 0 más), semiurgentes (realizar en 4 a 8 semanas) y urgentes (realizar en 4 semanas o menos). Aquellos urgentes son:

- Dolor torácico agudo y alta probabilidad de enfermedad arterial coronaria (EAC).

- Síndrome coronario crónico con alta probabilidad de eventos cardiacos mayores.

- Planeación de intervención estructural urgente.

- Evaluación de potenciales trombos intracavitarios.

- Miocardiopatía aguda, con baja a intermedia probabilidad clínica de EAC.

- Disfunción valvular protésica aguda y/o endocarditis, abscesos.

- Tumoración cardiaca maligna y planeación de biopsia o cirugía ${ }^{11}$.

El médico que interpreta el estudio debe evaluar los campos pulmonares para búsqueda de hallazgos sugestivos de infección por COVID-1912.

Respecto a la medicina nuclear si es posible, se prefiere la perfusión por PET, se debe buscar disminuir lo más posible la estancia de los pacientes en el servicio, hay que considerar usar protocolos de solo estrés en pacientes de bajo riesgo y corrección de atenuación para disminuir la estancia en el servicio, se prefiere el estrés farmacológico al físico por un menor riesgo de exposición a particulas; si se requiere esfuerzo físico se debe de proteger al personal con EPP ${ }^{13,14}$.
El estudio por resonancia magnética es infrecuente su uso de forma "urgente" en gran parte debido a la duración del mismo estudio, se sugiere diferir hasta nuevo aviso aquellos estudios que no afecten de manera significativa, la evolución clínica o la conducta terapéutica del mismo ${ }^{15,16}$.

\section{Tratamiento con IECA's y ARA-2}

Según la información obtenida en múltiples países se ha pensado en la probabilidad de que la hipertensión arterial sistémica puede estar asociada con un mayor riesgo de mortalidad en sujetos hospitalizados con infección por COVID-19 así mismo se ha considerado una relación de posibles efectos adversos de los inhibidores de la enzima convertidora de angiotensina o bloqueadores del receptor de angiotensina y que pueden aumentar tanto el riesgo de infección como la gravedad del SARS-CoV2, la preocupación surge de la observación en la manera similar al coronavirus que causa el SARS, el virus COVID-19 se une a una enzima específica llamada ACE2 para infectar células y los niveles de ACE2 aumentan después del tratamiento con inhibidores de la enzima convertidora de angiotensina o bloqueadores del receptor de angiotensina; esta idea sobre tratamiento con inhibidores de la enzima convertidora de angiotensina (IECA's) o bloqueadores del receptor de angiotensina (ARA2) en relación con COVID-19 no tiene una base científica sólida ni evidencia que lo respalde, de hecho, existe evidencia de estudios que sugiere que estos medicamentos podrían ser protectores contra complicaciones pulmonares graves en pacientes con infección por COVID-19 e incluso se publicó una revisión en China, donde los pacientes hipertensos que tomaban fármacos antihipertensivos se compararon si estos fueran IECA's/ARA2 versus tomar otros diferentes, mostrando en las conclusiones una reducción de la mortalidad intrahospitalaria del 9.8 a $3.7 \%$, sugiriendo un gran beneficio de tomar dichos medicamentos; deseamos hacer énfasis en que recomendamos a médicos y pacientes que continúen el tratamiento habitual con cualquier tipo de antihipertensivos incluyendo los antes mencionados.

Respecto a la cardiopatía isquémica, a nivel global en varios lugares se ha suspendido la atención de los pacientes isquémicos o se ha optado por un tratamiento menos invasivo, pero también menos efectivo. La intención ha sido disminuir el riesgo de contagio a los pacientes, familiares y personal hospitalario. Sin embargo, esta decisión enfrenta a los servicios médicos con varios dilemas: ¿es seguro?, ¿es ético?, ¿la 
seguridad de los equipos de atención está por arriba de la salud de los pacientes?, ¿cuánto tiempo es apropiado mantener este cambio?

\section{Síndromes coronarios}

Es necesario que los servicios médicos que reciban pacientes con cardiopatía isquémica los clasifiquen en dos grupos de acuerdo con la información disponible en el momento de su atención para limitar el contagio de otros pacientes, familiares, personal médico y hospitalario de apoyo, evitando poner en contacto o reunir pacientes sin infección con pacientes portadores:

1. Pacientes con sospecha de infección por SARSCoV-2 o con diagnóstico confirmado de COVID-19 y que se presentan con síntomas de isquemia miocárdica concurrente o con complicaciones cardiacas secundarias.

2. Pacientes habituales sin diagnóstico ni sospecha de infección por SARS-CoV-2, en quienes los síntomas de isquemia miocárdica son el motivo de atención médica.

Se debe interrogar presencia de fiebre, tos, dificultad respiratoria, medir la temperatura y saturación arterial de oxigeno en todo paciente antes de su traslado a sala de cateterismo.

Existe un acuerdo general en diferir los procedimientos invasivos, percutáneos o quirúrgicos, en los pacientes con SCCE (síndromes coronarios crónicos estables) durante la emergencia sanitaria actual. Los pacientes con SCCE durante el periodo de pandemia deben recibir tratamiento medico óptimo y ser advertidos de buscar atención de urgencia en caso de presentar síntomas de inestabilidad isquémica ${ }^{17}$.

En el contexto actual de la pandemia por COVID-19 no existe un acuerdo unánime para definir la mejor estrategia de tratamiento para los pacientes con un SICA. El resto del documento se enfoca en revisar la información disponible, acumulada en un periodo muy breve de tiempo, sobre las diferentes propuestas de tratamiento descritas, para después presentar nuestro posicionamiento y recomendaciones ${ }^{18}$.

Se deben considerar dos situaciones encaminadas a disminuir el riesgo de contagio. Uno, limitar el acercamiento y el contacto con los pacientes al realizar la exploración física y/o un ecocardiograma transtorácico, buscando obtener el máximo de información con el mínimo de contacto físico con el paciente y dos, se debe considerar el uso de la angiotomografía coronaria como una herramienta rápida y no invasiva para confirmar o excluir la presencia de enfermedad coronaria como causa de los síntomas cuando se considere adecuado. Los elementos fundamentales para el diagnóstico son síntomas sugestivos, un ECG 12 derivaciones y biomarcadores miocárdicos ${ }^{18}$.

Angiotomografía coronaria: en caso de duda razonada sobre un origen coronario de los síntomas y si existe la disponibilidad se debe considerar su uso. Agiliza el proceso de confirmación o descarte, acorta tiempo de estancia hospitalaria, disminuye el uso de insumos hospitalarios, la ocupación de salas de hemodinamia y la exposición al contagio del personal que participa en salas de cateterismo ${ }^{19}$.

En pacientes con diagnóstico de IAMCEST la intervención coronaria percutánea primaria (ICPp) es el método de elección para la reperfusión coronaria y se reserva la fibrinólisis (FL) para los casos en los que no está disponible el intervencionismo coronario percutáneo (ICP) o cuando el traslado del paciente a un hospital para intervencionismo significa un retraso de tiempo significativo. A raíz de la pandemia por COVID-19, han surgido posturas diferentes para tratar a los pacientes con IAMCEST, estas diferencias reflejan la situación específica que enfrentan las sociedades médicas al momento de elaborar sus recomendaciones ${ }^{20}$.

Un reporte y opiniones de líderes de opinión recomiendan el uso de FL como primera opción de reperfusión, tanto en pacientes sin contagio como en los que tienen sospecha o diagnóstico de COVID-19 y reservan la ICP para los pacientes con una FL fallida, siempre y cuando el beneficio sea mayor al riesgo, proponen que los pacientes que tienen neumonía grave por SARS-CoV-2, con signos vitales inestables y un IAMCEST concurrente reciban únicamente tratamiento médico de soporte, sin acceder a FL o ICPp, hasta que ocurra la recuperación de la neumonía ${ }^{21}$. Estas recomendaciones son sumamente restrictivas, cierran la opción de ICPp y limitan la opción del ICP de rescate solo para casos seleccionados ${ }^{22}$.

Múltiples sociedades médicas mantienen sus recomendaciones habituales durante la pandemia, los pacientes (con o sin sospecha de infección, con o sin COVID-19) con un IAMCEST requieren atención obligatoria y deben ser reperfundidos preferentemente por ICPp, en especial los que tienen angina persistente $y / 0$ compromiso hemodinámico; además mantienen la opción de ICP de rescate (ICPr). Aceptan que la FL puede ser una alternativa en pacientes con neumonía por SARS-CoV-2 que desarrollan un IAMCEST y se encuentran estables. La Sociedad Española de Cardiología, es contundente en reconocer que la ICPp debe seguir siendo la estrategia de reperfusión preferida en 
la mayoría de los pacientes y que la FL es una opción en los pacientes con neumonía grave y con dificultad para su movilización o traslado ${ }^{23}$. España es uno de los países con la cifra de contagios y tasa de mortalidad más alta en este momento de la pandemia, sus servicios de salud se encuentran sobrepasados y colapsados por la demanda excesiva de pacientes con COVID-19; en medio de esta crisis sanitaria su consenso es privilegiar la reperfusión del IAMCEST por ICPp ${ }^{24}$.

En general en los pacientes que se presentan con un IAMSEST 0 angina inestable se debe identificar el riesgo específico de complicaciones para decidir el mejor momento para ser llevados a cateterismo ${ }^{25}$. Independientemente de su riesgo, es indispensable conocer la anatomía coronaria en todos los pacientes y de acuerdo con los hallazgos evaluar el método de revascularización. Esta conducta habitual, al igual que ocurre con el IAMCEST, se ha modificado durante la pandemia y se replican varias de las posturas descritas para los SICA con elevación del segmento $\mathrm{ST}^{26}$.

\section{Recomendaciones de cirugía cardiaca y COVID}

Respecto a las recomendaciones de cirugía cardiaca, aunque todos estamos de acuerdo en aplazar las cirugías electivas, desafortunadamente, la cirugía electiva no esta claramente definida, y las necesidades y demandas son diferentes para las diversas subespecialidades quirúrgicas; y esto aunado a que la cirugía cardiaca ${ }^{27}$, no está en la primera línea de trabajo en la pandemia, y ante la probabilidad alta de las necesidades que se tendrán, la cirugía cardíaca se hará flexible ${ }^{28}$, y tendrá que adaptarse a esta postura de reprogramar la cirugía no urgente, intentando generar un equilibrio de los recursos, dados los desafíos potenciales que genera esta pandemia, con la necesidad de dispositivos médicos, en específico los ventiladores, ECMO o similares, socorridos en nuestra área quirúrgica y necesarios para solventar esta crisis ${ }^{8}$.

\section{Medidas para el personal de salud}

Este tipo de consideraciones, nos conduce a proponer una serie de medidas y recomendaciones, al personal que pudiera estar en posible contacto con esta enfermedad COVID-1929.

- Cancelar la consulta médica que no sea prioritaria bajo la directriz nacional de promover el autoaislamiento en casa y disminuir las concentraciones de personas en salas de espera que funcionan como focos de diseminación de la infección.

- Prohibir a los mayores de 60 años las visitas ambulatorias a las salas de cirugía.

- Minimizar los tiempos de intervención y de exposición del grupo de la salud, con el objetivo de disminuir el riesgo de contagio de personal, de realizarse algún procedimiento.

- Limitar el uso de camas en las unidades de cuidados intensivos, así como el uso de ventiladores.

- Llegada al hospital únicamente con el material indispensable para la realización de sus actividades.

- Eventualmente, utilizar las salas de cirugía como zonas de expansión de la unidad de cuidados intensivos en el contexto de una emergencia nacional.

- Limitar las reuniones médicas y adaptarlas a reuniones virtuales.

- Suministrar información veraz a todo el equipo, con fundamento en recomendaciones mundiales, basadas en la evidencia científica.

- Postergar cirugías electivas o complejas no urgentes que requieran varios días de unidad de cuidado intensivo.

- Intervenir pacientes con COVID-19 cuya cirugía sea de absoluta emergencia.

- Considerar que las recomendaciones abarcan a los pacientes, que una vez ingresados al hospital no pueden ser egresados del mismo, sin haber sido tratados ${ }^{30}$.

\section{Insuficiencia cardiaca}

Respecto a la insuficiencia cardiaca (IC), en pacientes ambulatorios, dado que la población con IC tiene un mayor riesgo de infección y peor pronóstico si cursan con COVID-19, sugerimos que es necesario el aislamiento social y trabajar desde casa, durante todo el tiempo de la emergencia sanitaria, procurar establecer consulta a distancia y limitar al máximo las visitas presenciales, durante la duración de la contingencia sanitaria, el apoyo de enfermería en el seguimiento es fundamental, así como complementar la visita con la obtención de los datos que el paciente pueda realizar en su domicilio (disnea, edema, presión arterial, peso, frecuencia cardiaca, saturación de oxígeno $\left(\mathrm{SaO}_{2}\right)$, temperatura, etc.), no cambiar o suspender el tratamiento de base, especialmente cuando la indicación es la mejoría pronóstica, como en el caso de la IC con fracción de expulsión reducida. Importante enfatizar en la continuidad del tratamiento con IECA/ARA2/Sacubitril-Valsartán y en el recomendado de acuerdo con guías; en 
caso de que el paciente, cuente con un dispositivo implantado (DAl, marcapasos, o resincronizador), procurar revisarlos por telemetría, si el paciente presenta datos de descompensación, procurar la atención en domicilio o en su defecto, utilizar la corta estancia u "hospital de día" para evitar la hospitalización ${ }^{31}$.

En caso de pacientes hospitalizados por descompensación de insuficiencia cardiaca, se deben buscar los objetivos de evitar el contagio del virus y mejorar la IC lo más pronto posible ${ }^{32}$. Para ello se requiere: planificar desde antes de su ingreso al hospital, la terapia a proporcionar, gestionar que su ingreso sea directo a hospitalización, preferentemente sin pasar por el servicio de urgencias, hospitalizarlo en zona libre de Covid-19 (establecido por cada unidad médica), permanecer en la habitación con un máximo de un acompañante y sin visitas, únicamente se realizarán exámenes de laboratorio o gabinete que se consideren imprescindibles para la estratificación y manejo del paciente, en cuanto sea factible, se deben plantear estrategias de alta temprana con soporte de terapias intermitentes mediante hospitalización a domicilio, visitas en domicilio o atención telefónica por parte del personal médico de insuficiencia cardiaca para disminuir el tiempo de estancia en zonas con alto riesgo de contagio y liberar camas de hospitalización, finalmente establecer el diagnóstico de sospecha de infección por Covid-19 ante condiciones clínicas 0 de laboratorio (NT proBNP, Dímero D, PCR de alta sensibilidad, Troponinas, entre otros $)^{33}$.

\section{Anticoagulación}

En relación a los pacientes con enfermedad por coronavirus-19 (COVID-19) con tratamiento con algún anticoagulante oral directo (ACOD): dabigatrán, rivaroxabán, apixabán o con un antagonista de la vitamina $\mathrm{K}$ (AVK), como warfarina o acenocumarol y requieran tratamiento para COVID-19, considerar sustituirlo por heparina de bajo peso molecular (HBPM); en pacientes con tratamiento anticoagulante previo que sean dados de alta con tratamiento antiviral, se recomienda no reiniciar el tratamiento oral y continuar con HBPM a dosis terapéuticas mientras continúe éste ${ }^{34}$. El tratamiento con antiagregantes no contraindica la tromboprofilaxis farmacológica con HBPM o heparina no fraccionada (HNF) o Fondaparinux en enfermos hospitalizados con COVID-19 moderada a grave. Si el paciente tiene indicación para antiagregación, ésta deberá continuarse y agregar la tromboprofilaxis farmacológica que se requiere de acuerdo a los factores de riesgo ${ }^{35}$.
Todos los pacientes que se hospitalicen por COVID-19 serán evaluados con escala de Padua para riesgo de ETV, también se evaluará el riesgo de hemorragia con la escala IMPROVE-Hemorragia. Si no hay contraindicación para tromboprofilaxis farmacológica, ésta se ajustará al peso del paciente, utilizando el medicamento que se encuentre disponible (HNF, HBPM, Fondaparinux). La tromboprofilaxis se administrará durante todo el periodo de hospitalización y al egreso se continuará por 7 dias. Todos los pacientes con COVID-19 que se hospitalicen y que tengan antecedentes de algún tipo de trombosis (evento vascular isquémico, infarto agudo del miocardio, enfermedad tromboembólica venosa, trombosis arterial periférica, trombofilia) seán considerados como de alto riesgo de trombosis y se dará tromboprofilaxis a dosis intermedias con HBPM (enoxaparina: $1 \mathrm{mg} / \mathrm{Kg} / \mathrm{día}$ ) o HNF en infusión continua a dosis suficientes para prolongar el tiempo de tromboplastina parcial activado (TTPa) de 2 a 3 veces el testigo); pacientes con ventilación mecánica o aquellos pacientes con diagnóstico o sospecha alta de tromboembolia pulmonar/trombosis venosa profunda, si no hay contraindicación, se deberá inciar anticoagulación a dosis de (1mg/Kg/cada $12 \mathrm{~h}$ en caso de enoxaparina $)^{36}$. En caso de terapia con clopidogrel o ticagrelor en pacientes que requieran tratamiento antiviral, se recomienda sustituirlos dadas las interacciones medicamentosas ${ }^{37}$. Hay que considerar las posibles interacciones de los anticoagulantes de acción directa y de los cumarínicos con los fármacos empleados para el coronavirus, como antirretrovirales o hidroxicloroquina. En especial, los anticoagulantes orales directos.

En aquellos pacientes NO COVID-19 que se encuentren en tratamiento anticoagulante con AVK (prótesis valvulares, fibrilación auricular valvular, síndrome antifosfolípido) y que requieren de vigilancia con INR, éste se deberá espaciar hasta un máximo de 12 semanas en aquellos pacientes que han mantenido un INR estable. Sugerimos una evaluación virtual o vía telefónica para el ajuste de la dosis de $\mathrm{AVK}^{38}$.

\section{Conclusiones y consideraciones}

Como conclusión, es importante enfatizar que debemos extremar las precauciones de protección tanto del personal de salud como de nuestros pacientes, para disminuir al máximo el número de contagios, en especial al grupo más vulnerable dentro de las cardiopatías que son los pacientes con cardiopatía isquémica, hipertensión arterial, IC, trasplantados y con HAP, tratando de resolver las dudas mediante comunicación telefónica o telemedicina. Cuando estos paciente se 
infectan por COVID-19, se puede agravar tanto por la misma condición cardiológica, como por la propia infección. Esperemos que médicos, farmacéuticas, sociedades médicas, gobierno y sociedad en general, en conjunto, podamos combatir de manera eficiente esta pandemia y ofrecer las mejores conductas de prevención y terapéuticas para el bienestar de los pacientes en este grupo especial de muy alto riesgo, como son los enfermos con patologías cardiovasculares, así como orientar sobre los cuidados y medidas de protección y actividad física, que sin duda ayudarán a prevenir complicaciones y mejorar sus espectativas de calidad de vida y mejor pronóstico.

\section{Responsabilidades éticas}

Protección de personas y animales. Los autores declaran que para esta investigación no se han realizado experimentos en seres humanos ni en animales.

Confidencialidad de los datos. Los autores declaran que han seguido los protocolos de su centro de trabajo sobre la publicación de datos de pacientes.

Derecho a la privacidad y consentimiento informado. Los autores declaran que en este artículo no aparecen datos de pacientes.

\section{Bibliografía}

1. Davies A, Thompson KA, Giri K, Kafatos G, Walker J, Bennett A. Testing the efficacy of homemade masks: Would they protect in an influenza pandemic? Disaster Med Public Health Prep. 2013;7(4):413-8.

2. Brienen, Nicole CJ, Timen A, Wallinga J, Van Steenbergen JE, Teunis $P$. The effect of mask use on the spread of influenza during a pandemic. Risk Anal. 2010;30(8):1210-8.

3. Van der Sande M, Teunis P, Sabel R. Professional and home-made fase masks reduce exposure to respiratory infections among the general population. PLoS One. 2008;3(7):e2618.

4. Maclntyre CR, Cauchemez S, Dayer DE, Seale H, Cheung P, Browne G, et al. Face mask use and control of respiratory virus transmisión in households. Emerg Infect Dis. 2009;15(2):233-41.

5. Interim Guidance for Healthcare Providers during COVID-19. Outbreak [Internet]. EE.UU.: American Heart Association, U.S. Centers for Disease Control, World Health Organization; 11/03/2020. Disponible en: https:// professional.heart.org/idc/groups/ahamah-public/@wcm/@sop/@smd/ documents/downloadable/ucm_505872.pdf

6. Anesthesia Patient Safety Fundation and World Federation of Societies of Anesthesiologist

7. COVID-19 Clinical Guidance for the Cardiovascular Care Team [Internet] EE.UU.: American College of Cardiology; 6 de marzo de 2020. Disponible en: https://www.acc.org// /media/Non-Clinical/Files-PDFs-Excel-MSWord-etc/2020/02/S20028-ACC-Clinical-Bulletin-Coronavirus.pdf

8. Wang D, Hu B, Hu C, Zhu F, Liu X, Zhang J, et al. Clinical characteristics of 138 hospitalized patients with 2019 novel coronavirus-infected pneumonia in Wuhan, China. JAMA. 2020 Feb 7. doi: 10.1001/jama.2020.1585. [Epub ahead of print]

9. García Fernández MA, Cabrera Schulmeyer MC, Azcárate Agüero PM. Documento sobre el uso de la ecocardiografía en pacientes con COVID-19 [Internet]. Sociedad Española de Imagen Cardiaca; 17/03/2020. Disponible en: https://ecocardio.com/docs/UsoEcocardiografiaCOVID19.pdf

10. Cook TM, El-Boghdadly K, McGuire B, McNarry F, Patel A, Higgs A. Consensus guidelines for managing the airway in patients with COVID-19: Guidelines from the Association of Anaesthetists, the Difficult Airway Society, the intensive Care Society, the Faculty of Intensive Care Medicine and the Royal College of Anaesthetists. Anaesthesia. 2020;75(6):785-99.

11. Kirkpatrick JN, Mitchell C, Taub C, Kort S, Hung J, Swaminathan M. ASE Statement on Protection of Patients and Echocardiography Service Pro- viders During the 2019 Novel Coronavirus Outbreak. J Am Coll Cardiol. 2020 Apr 6. doi: 10.1016/j.jacc.2020.04.002. [Epub ahead of print]

12. Choi AD, Abbara S, Branch KR, Feuchtner GM, Ghoshhajra B, Nieman K, et al. Society of Cardiovascular Computed Tomography guidance for use of cardiac computed tomography amidst the COVID-19 pandemic Endorsed by the American College of Cardiology. J Cardiovasc Comput Tomogr. 2020 Mar 21. doi: 10.1016/j.jcct.2020.03.002. [Epub ahead of print]

13. Kooraki S, Hosseiny M, Myers L, Gholamrezanezhad A. Coronavirus (COVID-19) outbreak: What the Department of Radiology should know. J Am Coll Radiol. 2020;17:447-51.

14. Revel MP, Parkar AP, Prosch H, Silva M, Sverzelatti N, Gleeson F, et al.; on behalf of the European Society of Radiology (ESR) and the European Society of Thoracic Imaging (ESTI). COVID-19 patients and the Radiology department - advice from the European Society of Radiology (ESR) and the European Society of Thoracic Imaging (ESTI). Eur Radiol. 2020 Apr 20. doi: 10.1007/s00330-020-06865-y. [Epub ahead of print]

15. Skali $\mathrm{H}$, Murthy VL, Al-Mallah $\mathrm{MH}$, Bateman TM, Beanlands R, Better $\mathrm{N}$ et al. Guidance and Best Practices for Nuclear Cariology Laboratories during the Coronavirus Disease 2019 (COVID-19) Pandemic: An Information Statement from ASNC and SNMMI [Internet]. American Society of Nuclear Cardiology; 2 de abril de 2020. Disponible en: https://zenodo. org/record/3738020\#.XoX62XIpBhF

16. SCMR's COVID-19 preparedness toolkit [Internet]. Society for Cardiovascular Magnetic Resonance; 25 de marzo de 2020. Disponible en: https:// scmr.org/page/COVID19

17. Welt FGP, Shah PB, Aronow HD, Bortnick AE, Henry TD, Sherwood MW, et al. Catheterization laboratory considerations during the coronavirus (COVID-19) pandemic: From ACC's Interventional Council and SCAI. J Am Coll Cardiol. 2020;75(18):2372-5.

18. Speciality guides for patient management during the coronavirus pandemic. Clinical guide for the management of cardiology patients during the coronavirus pandemic [Internet]. Reino Unido: NHS; 20/03/2020. Disponible en: https://www.england.nhs.uk/coronavirus/wp-content/uploads/sites/52/2020/03/specialty-guide-cardiolgy-coronavirus-v1-20-march.pdf

19. Yang S, Manjunath L, Willemink MJ, Nieman K. The role of coronary CT angiography for acute chest pain in the era of high-sensitivity troponins. J Cardiovasc Comput Tomogr. 2019;13(5):267-73.

20. Ibanez B, James S, Agewall S, Antunes MJ, Bucciarelli-Ducci C Bueno H, et al. 2017 ESC Guidelines for the management of acute myocardial infarction in patients presenting with ST-segment elevation. Rev Esp Cardiol (Engl Ed). 2017;70(12):1082.

21. Zeng J, Huang J, Pan L. How to balance acute myocardial infarction and COVID-19: the protocols from Sichuan Provincial People's Hospital. Intensive Care Med. 2020 Mar 11. doi: 10.1007/s00134-020-05993-9. [Epub ahead of print]

22. Romaguera R, Cruz-González I, Jurado-Román A, Ojeda S, Fernández-Cisnal $A$, Jorge-Pérez $P$, et al. Consideraciones sobre el abordaje invasivo de la cardiopatía isquémica y estructural durante el brote de coronavirus COVID-19. Documento de consenso de la Asociación de Cardiología Intervencionista y la Asociación de Cardiopatía Isquémica de la Sociedad Española de Cardiología. REC Interv Cardiol. 2020:2:112-7.

23. Roffi M, Patrono C, Collet JP, Mueller C, Valgimigli M, Andreotti F, et al. 2015 ESC Guidelines for the management of acutecoronary syndromes in patients presenting without persistent ST-segment elevation: Task Force for the Management of Acute Coronary Syndromes in Patients Presenting without Persistent ST-Segment Elevation of the European Society of Cardiology(ESC). Eur Heart J. 2016;37(3):267-315.

24. Mahmud E. The Evolving Pandemic of COVID-19 and Interventional Cardiology. Catheter Cardiovasc Interv. 2020 Apr 12. doi: 10.1002/ ccd.28894. [Epub ahead of print]

25. Keeley EC, Boura JA, Grines CL. Primary angioplasty versus intravenous thrombolytic therapy for acute myocardialinfarction: a quantitative review of 23 randomised trials. Lancet. 2003;361(9351):13-20.

26. Drake D, Morrow CD, Kinlaw K, De Bonis M, Zangrillo A, Sade RM; Cardiothoracic Ethics Forum. Cardiothoracic surgeons in pandemics: Ethical considerations. Ann Thorac Surg. 2020 Apr 9. doi: 10.1016/j. athoracsur.2020.03.006. [Epub ahead of print]

27. Ti LK, Ang LS, Foong TW, Ng BSW. What we do when a COVID-19 patient needs an operation: operating room preparation and guidance. Can J Anaesth. 2020 Mar 6. doi: 10.1007/s12630-020-01617-4. [Epub ahead of print]

28. Lauer SA, et al.The incubation period of coronavirus disease 2019 (COVID 19) from publicly report confirmed cases. Estimation and application. Ann Intern Med. 2020 Mar 10.

29. Chen N, Zhou M, Dong X, Qu J, Gong F, Han Y, et al. Epidemiological and clinical characteristics of 99 cases of 2019 novel coronavirus pneumonia in Wuhan, China: a descriptive study. Lancet. 2020;395:507-13.

30. Implicaciones de la pandemia por COVID-19 para el paciente con insuficiencia cardiaca, trasplante cardiaco y asistencia ventricular. Recomendaciones de la Asociación de Insuficiencia Cardiaca de la Sociedad Española de Cardiología [Internet]. Sociedad Española de Cardiología, Asociación de Insuficiencia Cardiaca; 2020. Disponible en: https://secardiologia.es/images/secciones/insuficiencia/Implicaciones_de_la_pande- 
mia_por_COVID-19 para_el_paciente con insuficiencia_cardiaca trasplante_cardiaco_y_asistencia_ventricular.pdf

31. Recomendaciones al Subsistema Nacional de Donación y Trasplantes sobre la infección asociada al SARS-CoV-2 (COVID-19) [Internet]. México: Centro Nacional de Trasplantes; 1 de abril de 2020. Disponible en: https://www.gob.mx/cenatra/articulos/recomendaciones-al-subsistema-nacional-de-donacion-y-trasplantes-sobre-la-infeccion-asociada-alsars-cov-2-covid-19-238147

32. Torres A, Rivera A, García A, Arias C, Saldarriaga C, Gómez E, et al. Evaluación y tratamiento de la insuficiencia cardiaca durante la pandemia de COVID-19: resumen ejecutivo [Internet]. Sociedad Colombiana de Cardiología \& Cirugía Cardiovascular, Revista Colombiana de Cardiología; abril de 2020. Disponible en: http://revcolcard.org/evaluacion-y-tratamiento-de-la-insuficiencia-cardiaca-durante-la-pandemia-de-covid-19-resumen-ejecutivo/

33. Guidance from the CCS COVID-19 Rapid Response Team and CCS Affiliate organizations [Internet]. Canadian Cardiovascular Society; 2020. Disponible en: https://www.ccs.ca/en/

34. Galie N, Humbert M, Vachiery JL, Gibbs S, Lang I, Torbicki A, et al. 2015 ESC/ERS Guidelines for the diagnosis and treatment of pulmonary hypertension: The Joint Task Force for the Diagnosis and Treatment of Pulmonary Hypertension of the European Society of Cardiology (ESC) and the European Respiratory Society (ERS). Endorsed by: Association for European Paediatric and Congenital Cardiology (AEPC), International Society for Heart and Lung Transplantation (ISHLT). Eur Heart J. 2016;37(1):67-119.

35. Benza RL, Gomberg-Maitland M, Elliott CG, Farber HW, Foreman AJ, Frost $A E$, et al. Predicting survival in patients with pulmonary arteria hypertension: The REVEAL Risk Score Calculator 2.0 and comparison with ESC/ERS-based risk assessment strategies. Chest. 2019;156(2): 323-37.

36. Humbert M, Sitbon O, Yaici A, Montani D, O'Callaghan DS, Jais X, et al Survival in incident and prevalent cohorts of patients with pulmonary arterial hypertension. Eur Respir J. 2010;36(3):549-55

37. Pandolfi R, Barreira B, Moreno E, Lara-Acedo V, Morales-Cano D, Martinez-Ramas A, et al. Role of acid sphingomyelinase and IL- 6 as media- tors of endotoxin-induced pulmonary vascular dysfunction. Thorax. 2017;72(5):460-71

38. Pan IZ, Carey JR, Jacobs JA, Dechand J, Sessions JJ, Sorensen T, et al. Transitioning between prostanoid therapies in pulmonary arterial hypertension. Front Med (Lausanne). 2020;7:81.

\section{Lecturas recomendadas}

a. Hoeper MM, Benza RL, Corris P, de Perrot M, Fadel E, Keogh AM, et al. Intensive care, right ventricular support and lung transplantation in patients with pulmonary hypertension. Eur Respir J. 2019;53(1).

b. https://www. who.int/publications-detail/clinicalmanagement-of-severe-acute-respiratory-infection-when-novel-coronavirus-(ncov)-infection-is-suspected).

c. Padang R, Chandrashekar N, Indrabhinduwat M, Scott CG, Luis SA, Chandrasekaran $\mathrm{K}$, et al. Aetiology and outcomes of severe right ventricular dysfunction. Eur Heart J. 2020;41(12):1273-82.

d. Campo A, Mathai SC, Le Pavec J, Zaiman AL, Hummers LK, Boyce D et al. Outcomes of hospitalisation for right heart failure in pulmonary arterial hypertension. Eur Respir J. 2011;38(2):359-67.

e. Wang D, Li S, Jiang J, Yan J, Zhao C, Wang Y, et al. Section of Precision Medicine Group of Chinese Society of Cardiology, Editorial Board of Chinese Journal of Cardiology, Working Group of Adult Fulminant Myocarditis (2019) Chinese society of cardiology expert consensus statement on the diagnosis and treatment of adult fulminant myocarditis. Sci China Life Sci 62(2):187-202.https://doi.org/10.1007/s1142 7-018-9385-3.

f. Piotr Ponikowski, Adriaan A Voors, Stefan D Anker, Héctor Bueno, John G F Cleland, Andrew J S Coats, et al. ESC Scientific Document Group, 2016 ESC Guidelines for the diagnosis and treatment of acute and chronic heart failure: The Task Force for the diagnosis and treatment of acute and chronic heart failure of the European Society of Cardiology (ESC). Developed with the special contribution of the Heart Failure Association (HFA) of the ESC, European Heart Journal, Volume 37, Issue 27, 14 July 2016, Pages 2129-200, https://doi.org/10.1093/eurhearti/ehw128. g. https://www.youtube.com/watch?v=10_LAsOQExw 